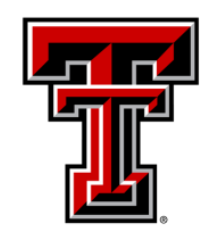

TEXAS TECH UNIVERSITY

Libraries"

\title{
MODEL EVALUATIONS OF SURFACE MODIFICATION BY ENERGETIC INCIDENT CARBON ATOMS ON GRAPHENE COATED COPPER ELECTRODES
}

\section{The Texas Tech community has made this publication openly available. Please share how this access benefits you. Your story matters to us.}

\begin{tabular}{|l|l|}
\hline Citation & $\begin{array}{l}\text { X. Qiu, J. Mankowski, J. C. Dickens, A. A. Neuber, and R. P. Joshi, } \\
\text { "Model Evaluations of Surface Modification by Energetic Incident } \\
\text { Carbon Atoms on Graphene Coated Copper Electrodes," Physics of } \\
\text { Plasmas 26, 013501 (2019). https://doi.org/10.1063/1.5056766 }\end{array}$ \\
\hline Citable Link & $\underline{\text { https://hdl.handle.net/2346/86908 }}$ \\
\hline Terms of Use & $\begin{array}{l}\text { This article may be downloaded for personal use only. Any other } \\
\text { use requires prior permission of the author and AIP Publishing. }\end{array}$ \\
\hline
\end{tabular}




\section{Model evaluations of surface modification by energetic incident carbon atoms on graphene coated copper electrodes}

Cite as: Phys. Plasmas 26, 013501 (2019); https://doi.org/10.1063/1.5056766

Submitted: 13 September 2018 . Accepted: 17 December 2018 . Published Online: 07 January 2019

(D) X. Qiu, (D) J. Mankowski, (D) J. C. Dickens, (D) A. A. Neuber, and (D) R. P. Joshi
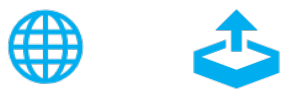

\section{ARTICLES YOU MAY BE INTERESTED IN}

Dynamic analysis of material ejection from cathodic metal nano-tips due to local heating and field generated stress

Physics of Plasmas 25, 022109 (2018); https://doi.org/10.1063/1.5018441

Calculations of secondary electron yield of graphene coated copper for vacuum electronic applications

AIP Advances 8, 015325 (2018); https://doi.org/10.1063/1.5019360

Probing changes in secondary electron yield from copper electrodes due to surface defects and changes in crystal orientation

Journal of Applied Physics 126, 123301 (2019); https://doi.org/10.1063/1.5113642 


\title{
Model evaluations of surface modification by energetic incident carbon atoms on graphene coated copper electrodes
}

Cite as: Phys. Plasmas 26, 013501 (2019); doi: 10.1063/1.5056766

Submitted: 13 September 2018 - Accepted: 17 December 2018 • Published Online: 07 January 2019

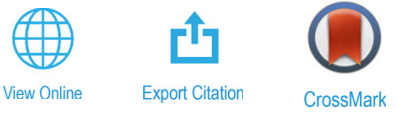

X. Qiu, (D) J. Mankowski, (D) J. C. Dickens, (D) A. A. Neuber, (D) and R. P. Joshi ${ }^{\text {a) }}$ (D)

\author{
AFFILIATIONS \\ Department of Electrical and Computer Engineering, Texas Tech University, Lubbock, Texas 79409, USA
}

a) Author to whom correspondence should be addressed: ravi.joshi@ttu.edu

\begin{abstract}
Thin nanoscale coating of metal electrodes by graphene promises to be a useful approach for suppressing the secondary electron yield and potential multipactor. Recent calculations showed reductions by as much as 50\% for graphene over copper electrodes for energies below $125 \mathrm{eV}$, with results in good agreement with experimental data. Here, the resistance to possible degradation of this structure, in response to incoming atomic projectiles, is gauged based on molecular dynamics simulations. Our results for surface irradiation by carbon atoms (as an example) on nanoscale graphene coatings indicate a defect threshold of about $35 \mathrm{eV}$, lower surface damage for thicker layers, negligible sputtering, and defects less than $6 \AA$ in dimension for energies up to 300 eV. The electrode structure is shown to be robust with better resistance to damage than metal alone.
\end{abstract}

Published under license by AIP Publishing. https://doi.org/10.1063/1.5056766

\section{INTRODUCTION}

The next generation of nuclear reactors and related structural materials needs to endure increased neutron doses for long periods of time without failure. ${ }^{1,2}$ Additionally, long-term radiation exposure is also a challenge to the survival of electronics on spacecraft. ${ }^{3}$ Hence, from the reliability standpoint, it is important to improve or at the very least gauge the radiation tolerance of materials and understand their effects. ${ }^{4-6}$ Towards this end, the present contribution is an attempt to gauge and understand surface damage in graphene coated copper electrodes. This composite material system has recently been shown to be effective at suppressing secondary electron emission (SEE) ${ }^{7-11}$ that can be produced by the bombardment of externally charged particles. Secondary electron emission (SEE) is known to be a major cause of performance degradation in various electronic devices, and the negative effects include the electron cloud phenomenon in accelerators ${ }^{12,13}$ and multipactor driven by radio frequency excitations. ${ }^{14-18}$ Greater losses such as potential outgassing from surfaces, ionization breakdown, and electrostatic discharges on the outer surface of satellites, leading to mission failures, ${ }^{19}$ are some of the consequences of SEE in high power devices. Additional advantages of using graphene for electrodes is that no post-treatment or ion-sputtering is required and the material has exceptional material strength and couples high electrical and thermal conductivities. ${ }^{20-22}$ In any case, surface damage in the present situation could lead to (i) graphene erosion which would increase secondary electron emission and (ii) cause nanoscale surface cracks and pits which could enhance local electric fields, thus promoting electron emission. These typically are the first stages of possible plasma formation.

Despite the promise, the reliability and durability of graphene coated system electrodes in harsh environments have not been probed in detail. Hence, the dependability and lifetime of such strategies that might utilize graphene coated materials remain unclear for the more rugged and demanding applications, such as space satellites. A potentially useful way forward, short of actual experimentation under a wide variety of conditions, is to simulate the response and quantify irradiatedinduced damage. Furthermore, unlike bulk materials for which estimates of irradiation-induced defects can be obtained based on the binary-collision approximations that combine statistical energy transfers to the host material, ${ }^{23-25}$ such Monte Carlo type methods are not directly applicable to low-dimensional systems as finite-size and many-body effects become important. Since the graphene coating is primarily responsible for SEE 
suppression, the focus here is on the first few monolayers at the surface. Thus, effects deep within the copper electrode such as possible displacement cascades $^{26}$ become irrelevant. Furthermore, since the current interest is driven by the reliability of high pulsed power devices, the energy range of interest is much smaller as compared to the hundreds of $\mathrm{keV}$ levels encountered in nuclear reactors. Also, since the process of multipactor arises in the context of radio-frequency (RF) excitations, the energy gain by charged particles in the system is typically not very high.

In this contribution, we simulate the effects of the impact by energetic atoms incident onto graphene layers grown on metal by using molecular dynamics. ${ }^{26-28}$ As an example, carbon atoms were chosen for the incident bombardment, while copper (a common electrode material) was used for the metal. The choice of carbon bombardment is loosely based on two considerations: (i) heavier elements would tend to do more damage and hence could be used to benchmark potential damage for worst case scenarios. However, the lighter elements are more common in the atmosphere, especially higher altitudes. In this regard, carbon represents a compromise and so was chosen. (ii) Since graphene processing often involves carbon implantation, ${ }^{29}$ the use of this element here serves the indirect benefit of yielding useful information for possible processing applications. Given the relatively small thickness of the graphene (relative to the underlying copper substrate) and its two-dimensional nature, bulk aspects such as defect propagation or the role of grain boundaries and dislocations were not considered. The present aim is merely to probe the utility of graphene layers as a surface coating for better shielding and structural robustness. It may also be mentioned that most previous calculations for damage in graphene have been based on free-standing sheets. ${ }^{30-32}$ Although relatively simple to implement, such stand-alone material scenarios lack a strong practical relevance. In any event, the primary aims of the present simulations were to (i) identify the types and abundance of defects in graphene upon irradiation by carbon atoms of varying energies, (ii) determine whether any threshold might exist and if so the minimal value of the incident energy, (iii) the energy-dependent transmission, reflection, and absorption coefficients through the graphene coated material, (iv) evaluations of any differences arising from changes in the graphene layer thicknesses, and (v) obtaining quantitative estimates of the damage size and area at various incident energies.

\section{MODEL DETAILS}

The molecular dynamics (MD) technique essentially involves solving the equations of motion for interacting particles numerically, subject to appropriate initial and boundary conditions. ${ }^{33}$ Here, LAMMPS, a classical MD simulator, ${ }^{34}$ was used. The interactions between the copper atoms in the metal were modelled based on the many-body Embedded-Atom Method (EAM). Compared to pair potentials, EAM provides a better description on metallic bonds and includes both the pairpotential energy term and the embedding energy from the surrounding atoms. ${ }^{35}$ A consistent set of embedding functions and short-range repulsive pair interactions for the facecentered-cubic metals (such as copper) is available to LAMMP users based on the data by Foiles et al. ${ }^{36}$ In describing the interactions between copper and the graphene carbon atoms, the 12-6 Lennard-Jones (LJ) type of van der Waals interactions was used. ${ }^{37}$ The LJ potential has been used in similar other simulations of cascade collisions in metal-graphene nanocomposites. ${ }^{38-40}$ Values for the LJ potential parameters were taken to be $\sigma=3.225 \AA$ and $\varepsilon=0.019996 \mathrm{eV}$ after Huang et al. ${ }^{40}$ Finally, the Adaptive Intermolecular Reactive Empirical Bond Order (AIREBO) Potential (which incorporates both the repulsive and attractive pair potentials), developed by Stuart et al. ${ }^{41}$ was used for the carbon interactions.

The simulation cell was taken to have a copper base (i.e., the substrate) on which one or more graphene monolayers were placed along the vertical $(z-)$ direction. The substrate dimensions were taken to be $4.95 \mathrm{~nm} \times 8.5 \mathrm{~nm} \times 4.9 \mathrm{~nm}$ along the $x-, y^{-}$, and $z$-directions. Since the aim was primarily geared towards the shielding effect that graphene layers can provide, and not any bulk effects, we deliberately did not choose a large substrate. Periodic boundary conditions were imposed along the lateral $(x$ and $y$ ) directions. The entire system was initially relaxed (prior to any bombardment) by running the LAMMPS simulations up to 200 ps without any forces, to achieve thermodynamic equilibrium. The NVE conditions were used.

\section{RESULTS AND DISCUSSION}

The results of our MD simulations obtained for one monolayer of graphene on copper are shown in Fig. 1. The probabilities of absorption, reflection, and transmission are plotted as a

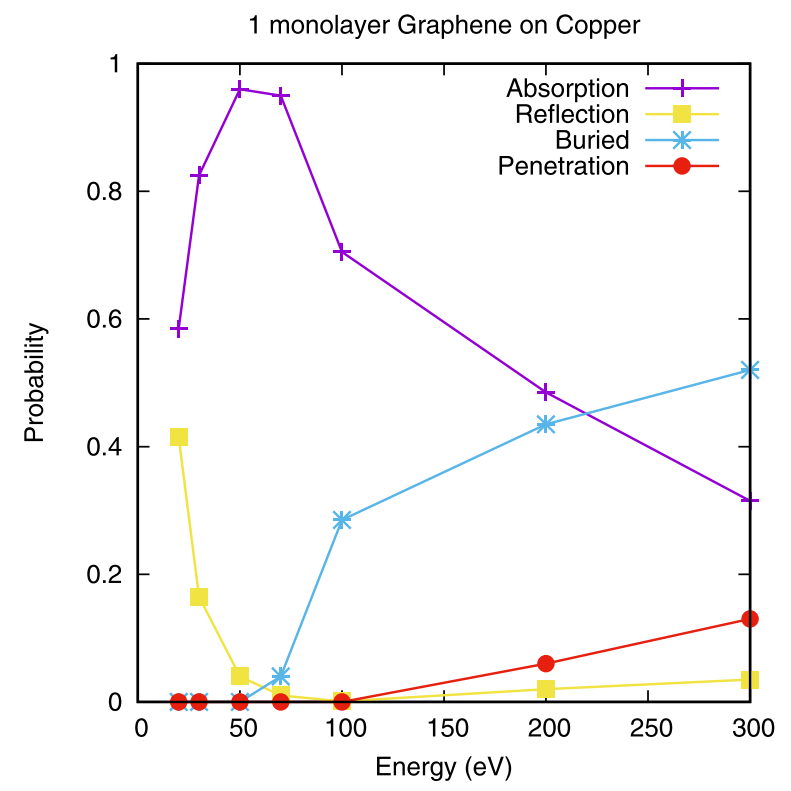

FIG. 1. MD simulation results obtained for one monolayer of graphene on copper. The probabilities of absorption, reflection, and transmission are plotted as a function of incident carbon atom energy. 
function of incident carbon atom energy. Overall, 1000 simulation runs were carried out at each energy, and the statistical response was gathered from the aggregated results. The absorption curve denotes incoming atoms getting absorbed by the graphene sheet, while the "Buried" curve refers to the incident particles getting buried in the copper substrate. Finally, "Penetration" alludes to the incident carbon going through the entire graphene-on-copper system. At low energies, the incident atom is mostly absorbed by the graphene, with reflection predicted for a small fraction. At values below $100 \mathrm{eV}$, there is no penetration through the nanocomposite system, although some incoming atoms would end up in the copper substrate. It is also clear that at higher energies, most incident atoms would not be in the graphene layer.

A more pertinent parameter is the damage caused by the incoming particles. The MD simulation result of Fig. 2(a) shows the damage probability within graphene as a function of incident energy. Almost no damage is predicted up to about $35 \mathrm{eV}$. While the probability in Fig. 2(a) rises to values as high as $82 \%$ at higher energies, an important aspect not conveyed by the probability plot is the relatively negligible scale of the damage. Most of the damage was predicted to have small areal dimensions, although it did get counted for the curve of Fig. 2(a). The dependence of the average defect size on the incident carbon atom energy is shown in Fig. 2(b). No defect creation or damage is predicted for energies below about $35 \mathrm{eV}$. Furthermore, the average lateral dimension of the damage at the highest $300 \mathrm{eV}$ energy is predicted to be roughly $6 \AA$. The curve of Fig. 2(b) for the average damage size (in $\AA^{2}$ ) can roughly be fit by the following energydependent quadratic: $f(E)=-2.474927 \times 10^{-4} E^{2}+0.1753922$ $\mathrm{E}+6.3$, with $\mathrm{E}$ being the incident energy in $\mathrm{eV}$. This sub-linear defect-size dependence on incident energy is similar to the result by Osetsky in copper. ${ }^{42}$ Essentially, defects are created via primary knock-on, which can potentially lead to cascades if the incident energies are high. However, for the present case of relatively modest energies, cascades do not arise. Instead, any defect or vacancy created is healed in part by the movement of neighboring atoms. Besides, the graphene atoms with their twodimensional structure can easily slide and move. This results in lowering the defect size and imparts a sub-linear energy dependence. The results of Fig. 2(b) roughly follow the trend first discussed by Robinson. ${ }^{43}$ Not much damage was predicted in the copper, which is consistent with rapid healing in metals. ${ }^{44}$ Essentially, the interaction process of incoming projectiles in metals consists of a ballistic period followed by a recombination (or healing) phase. The former lasts a few tenths of picoseconds, during which the primary particle can initiate numerous atomic displacements. This is followed by thermalization and follow-up recombinations, which cause the displaced atoms to recombine and readjust their configuration by moving to partially vacant lattice sites.

Calculations were also performed for two, three, and four graphene layers on copper, and the results are shown in Fig. 3. The absorption probability of Fig. 3(a) increases with the number of graphene layers as there is a greater material for the incoming atoms to be absorbed. The relatively higher reflection at low
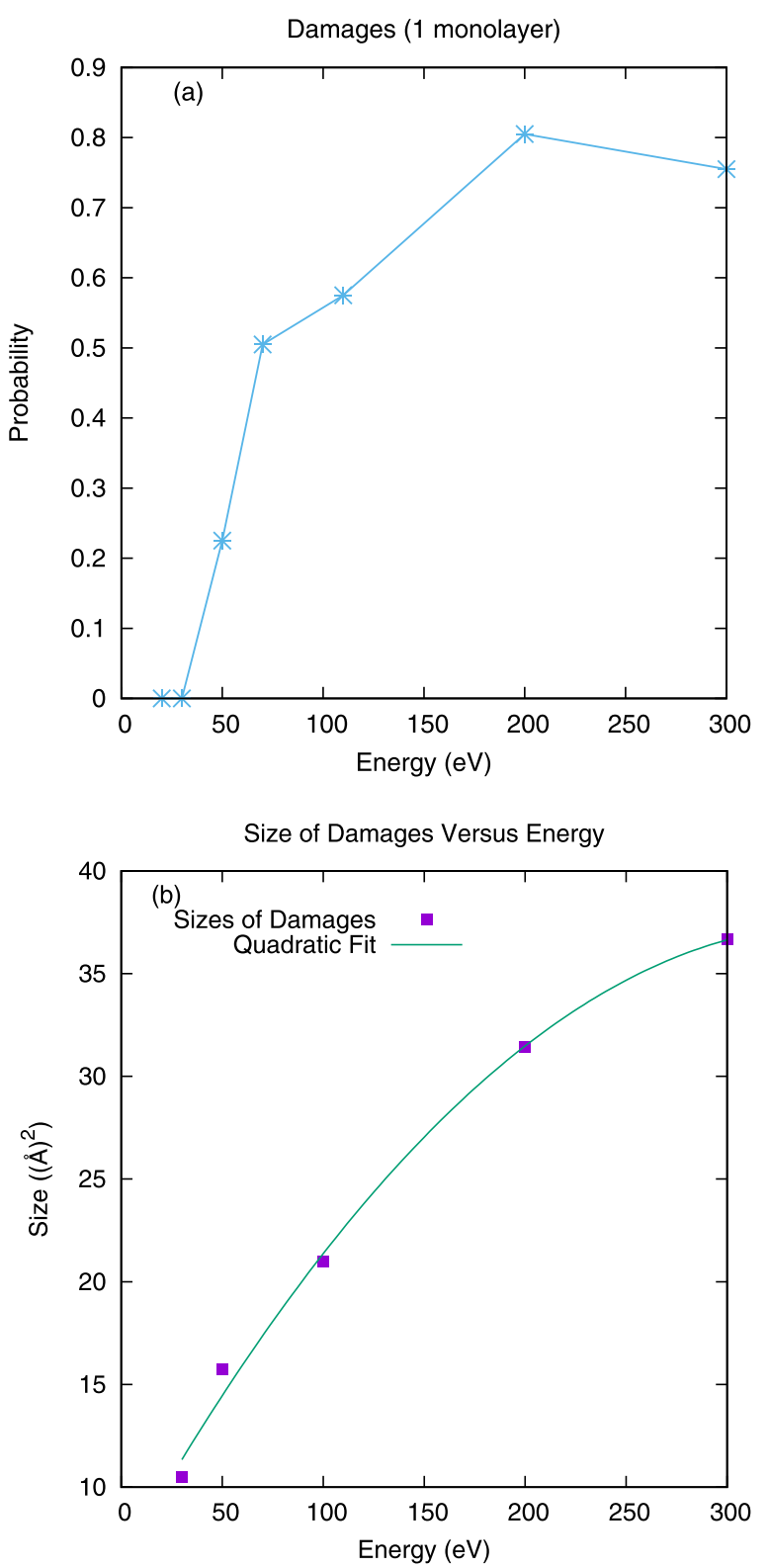

FIG. 2. Simulation results for damage in a monolayer of graphene on copper. (a) The probability of damage within graphene shown as a function of incident energy. (b) The dependence of average damage size on the incident energy.

energies and the larger penetration at higher energies give rise to local maxima for each absorption curve. The transmission curves of Fig. 3(b) show a monotonic increase with energy and a threshold effect. More interestingly, the sputtering probabilities given in Fig. 3(c) show that for energies around $300 \mathrm{eV}$, the sputtering yield for graphene coated copper is about $50 \%$. The largest yield is predicted to occur for a one-layer graphene structure, while increasing the number of sheets leads to a protective shielding effect. Thus, thicker coatings with at least three 

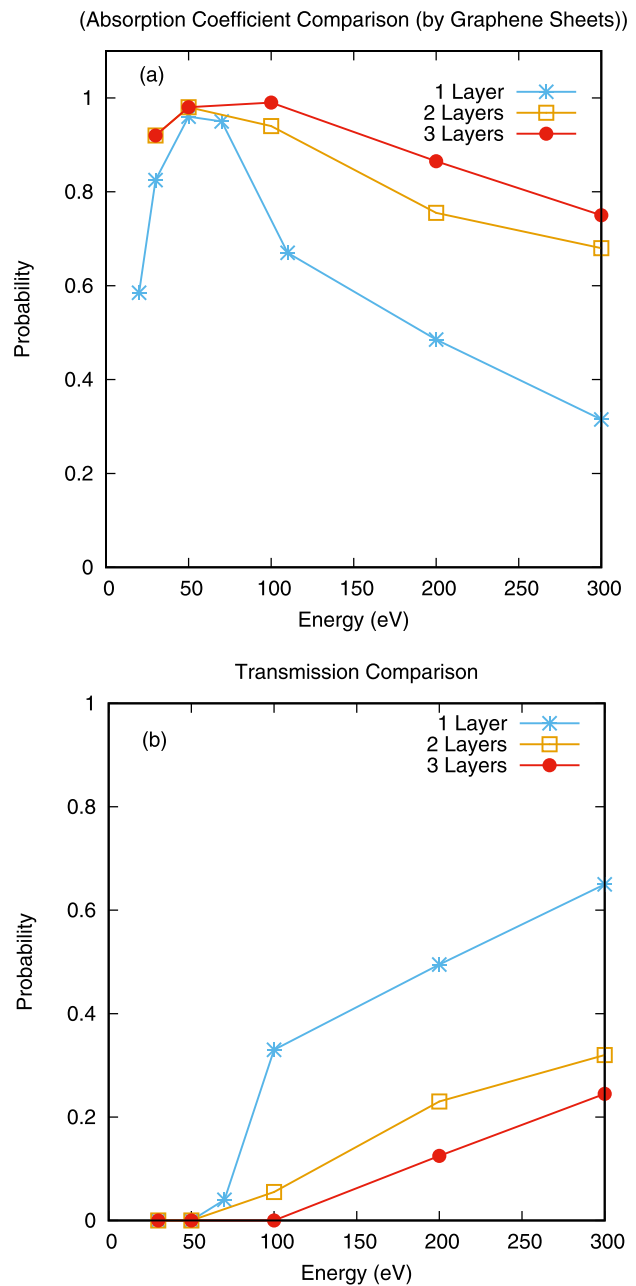

Sputtering Yield (Pure Copper and Graphene-on-Copper)

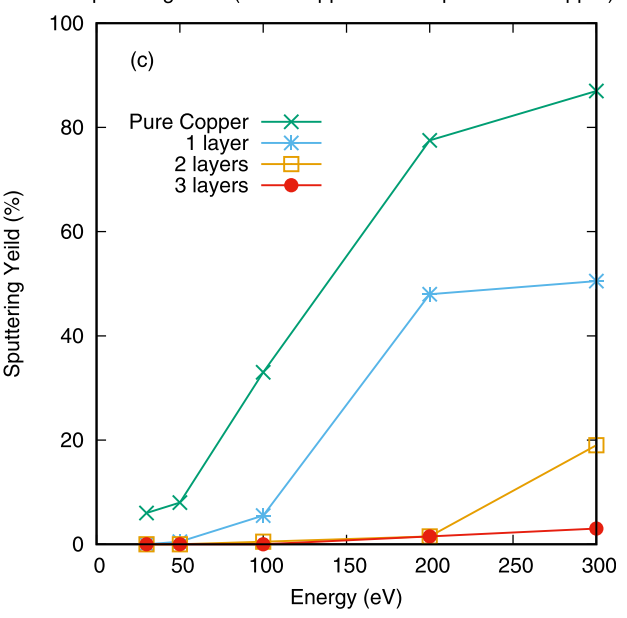

FIG. 3. Comparisons of the responses to incident carbon atoms of varying energies on nanocomposites with a different number of graphene layers on copper. (a) Absorption coefficient results, (b) transmission probabilities from MD calculations, and (c) energy dependence of the sputtering yield. or more monolayers would appear to be a more useful strategy to reduce damage to the topmost layer. This aspect of thicker layers is discussed subsequently. More interestingly, the sputtering yield for the copper material alone, without any graphene coatings, is predicted to be the highest. This is a clear implication then that graphene coatings, in addition to providing the benefit of reduced secondary electron emissions, ${ }^{11}$ would also offer a more stable and durable system against degradation.

Snapshots obtained from MD simulations showing the atomic configurations are shown in Fig. 4 and provide a more relevant picture of the impact on defect formation. A total of 1000 simulations were performed for each of the results given in Figs. 4(a)-4(c), and two layers of graphene were used. Due to the statistical variability, the plots given here should be viewed as qualitative indicators. Overall, two processes appear to be operative in the process of defect production due to incident atomic collisions with the graphene-on-copper system. Of these, some are the result of direct collision with graphene (and can be named "direct defects"), while atoms sputtered from other locations in the nanocomposite material system can also move and create indirect defects. Our MD simulations indicate the direct defects to mainly be mono-vacancies, while the indirect defects contribute to mono-vacancies and the formation of double- and poly-vacancies. With regard to actual results, the top view following irradiation from incident carbon atoms with an energy of $300 \mathrm{eV}$ is shown in Fig. 4(a). The red atoms denote graphene, the yellow area represents the underlying copper atoms, while the blue atoms [not seen in Fig. 4(a)] indicate the incident carbon. As seen from Fig. 4(a), a few isolated yellow patches denote local voids due to damage from the incoming carbon atoms. Overall, the sizes are not very large, and it is conceivable that the damage could self-heal upon subsequent local heating. Although such an analysis for surface re-construction could be carried out based on atomistic simulations, this self-healing aspect was not probed here. A snapshot at a lower energy of $150 \mathrm{eV}$ from the many simulation runs is shown in Fig. 4(b). Some areas of damage are evident, but the areal sizes are not very large. Finally, Fig. 4(c) shows the top view of the two graphene layers on copper with the carbon atoms incident at $50 \mathrm{eV}$ energy. An embedded carbon can be seen in blue in Fig. 4(c). The areal damage is predicted to be modest.

The results for different graphene thicknesses are also presented and briefly discussed for completeness. Figure 5 shows the MD results of the top view for copper with a single graphene layer. The structure following irradiation by carbon atoms of energies $300 \mathrm{eV}, 100 \mathrm{eV}$, and $50 \mathrm{eV}$ is given in Figs. 5(a)-5(c), respectively. An embedded carbon (shown in blue) can be seen in each figure. It becomes apparent that the damage can be larger than the previous 2-layer case of Fig. 4. As an example, for the $300 \mathrm{eV}$ case, more damage and distortions are seen in Fig. 5(a) as compared to Fig. 4(a). Next, the responses of copper coated with three and eight layers of graphene were obtained and are shown in Figs. 6 and 7. Figure 6 shows the side view of the atomic configuration of copper capped by a three-layer graphene coating. Fairly moderate levels of destruction and atomic displacements can be seen. On the other hand, for the eight- 

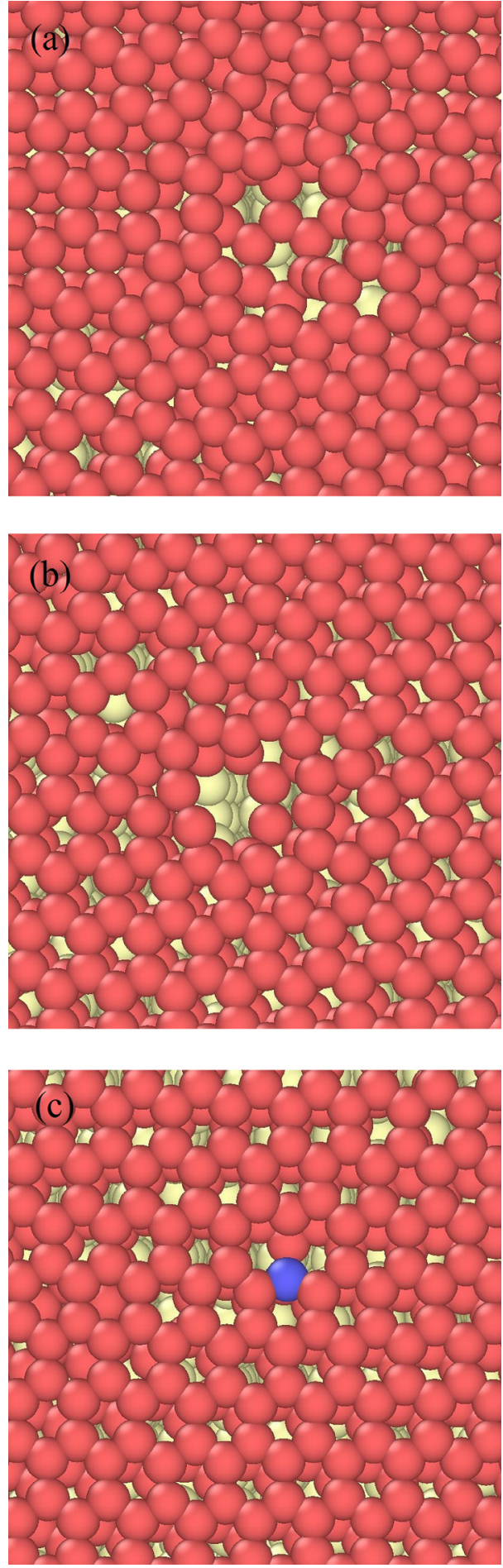

FIG. 4. MD snapshots showing the top view of the atomic placement and configuration for two graphene layers on copper following irradiation by carbon atoms of different energies. (a) $300 \mathrm{eV}$ atoms, (b) $150 \mathrm{eV}$ atoms, but with the scale slightly enlarged, and (c) $50 \mathrm{eV}$ atoms showing an embedded carbon in blue and one minor defect on the top right.
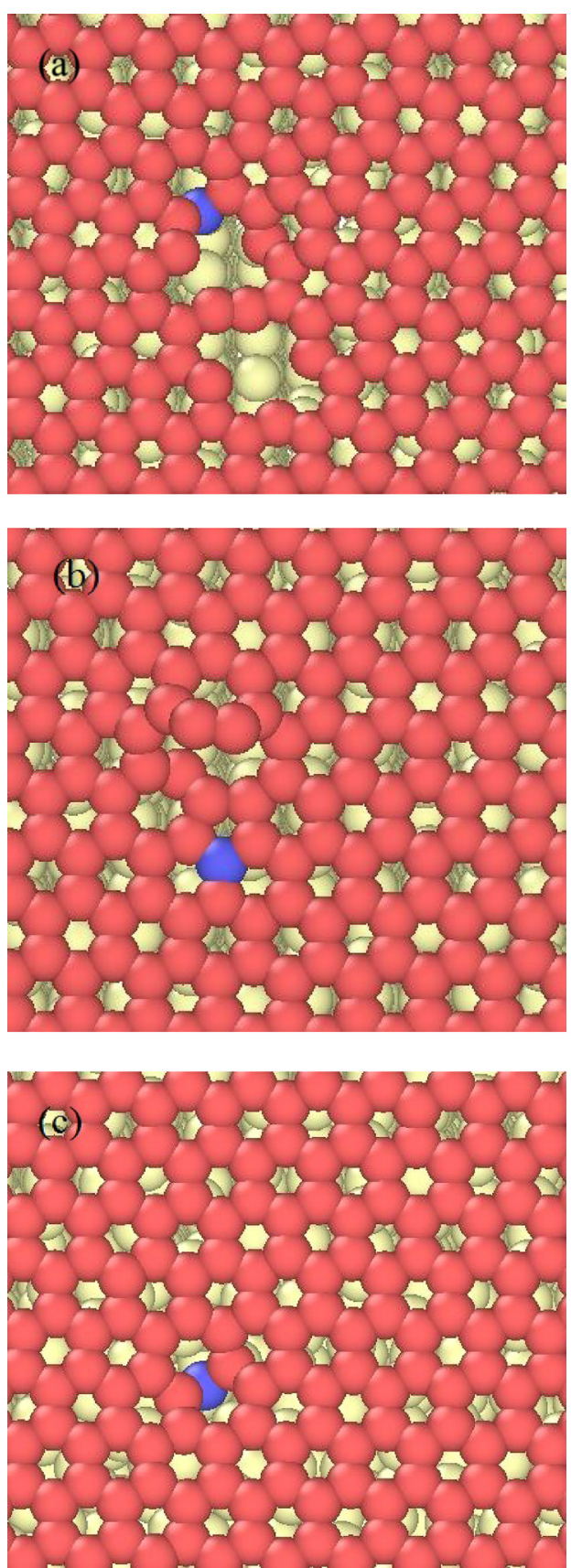

FIG. 5. MD snapshots showing the top view for copper with a single graphene layer, following irradiation by carbon atoms of energies: (a) $300 \mathrm{eV}$, (b) $100 \mathrm{eV}$, and (c) $50 \mathrm{eV}$. An embedded carbon in blue can be seen in each figure. The damage is seen to be more than the previous 2-layer case of Fig. 4.

layer structure of Fig. 7, the damage is seen is to be much less than the previous 3-layer case and the graphene sheets near the substrate and the copper itself remain virtually undamaged and defect free. 


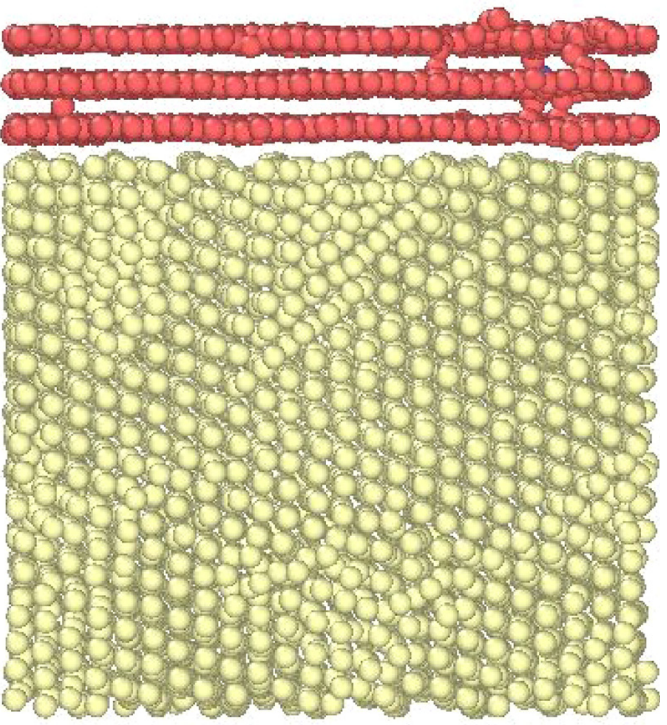

FIG. 6. Side view of the atomic configuration of copper capped by a three-layer graphene coating. Fairly moderate levels of destruction and atomic displacements can be seen.

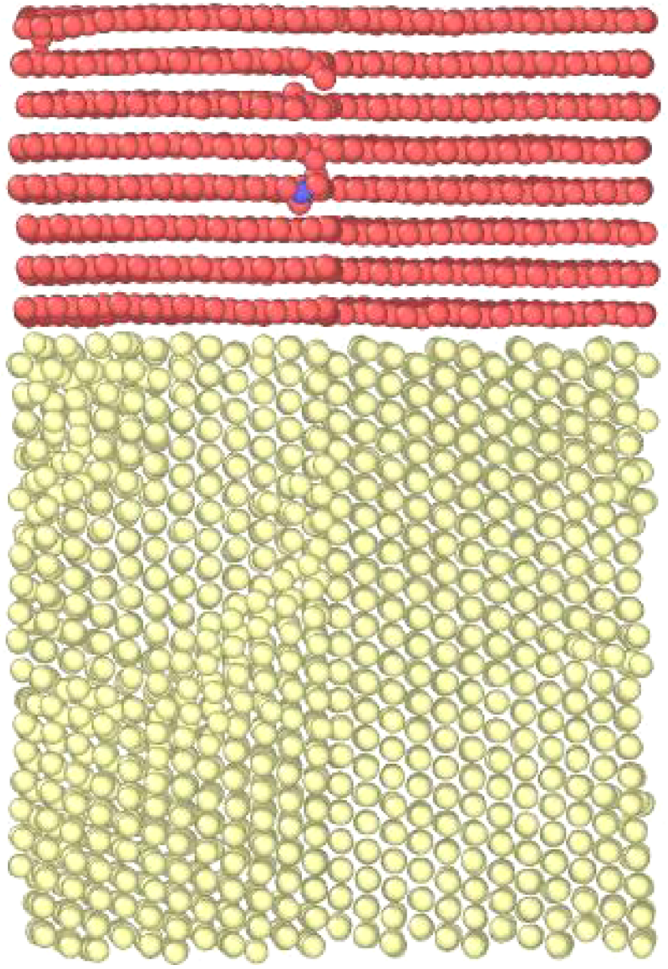

FIG. 7. Side view of the atomic configuration with eight layers of graphene on copper. The damage is seen is to be much less than the previous 3-layer case of Fig. 6, while the copper remains virtually undamaged and defect free.

\section{CONCLUSIONS}

In summary, the robustness and resistance to possible degradation of graphene coated copper in response to incoming carbon atom projectiles were gauged. The choice of this composite, comprising a few monolayers of graphene on copper, was driven by its potential to suppress secondary electron emission. Our results predict that the graphene coated composite would offer greater durability with lower sputtering damage as compared to copper alone. Simulations also indicate a defect threshold of about $35 \mathrm{eV}$, lower surface damage for thicker layers, negligible sputtering, and defects less than $6 \AA$ in dimension for incident energies up to $300 \mathrm{eV}$. It is even likely that minor damage could potentially self-heal or that surface reconstruction could occur, ${ }^{45}$ upon appropriate laser heat treatment. These aspects can be probed further but are beyond the present scope. Finally, multi-layer configurations are predicted to be helpful in further reducing sputtering or damage to the top layer as compared to copper alone.

\section{ACKNOWLEDGMENTS}

This research was supported in part by the Department of Defense MURI Grant No. FA9550-18-1-0062 on "Multipactor and Breakdown Susceptibility and Mitigation in Space-Based RF Systems" and the Office of Naval Research (N00014-18-1-2382).

\section{REFERENCES}

${ }^{1}$ S. J. Zinkle and J. T. Busby, Mater. Today 12, 12 (2009).

${ }^{2}$ I. J. Beyerlein, A. Caro, M. J. Demkowicz, N. A. Mara, A. Misra, and B. P. Uberuaga, Mater. Today 16, 443 (2013).

${ }^{3}$ E. G. Fu, M. Caro, L. A. Zepeda-Ruiz, Y. Q. Wang, K. Baldwin, E. Bringa, M. Nastasi, and A. Caro, Appl. Phys. Lett. 101, 191607 (2012).

${ }^{4}$ X. Bai, A. F. Voter, R. G. Hoagland, M. Nastasi, and B. P. Uberuaga, Science 327, 1631 (2010).

${ }^{5}$ I. J. Beyerlein, M. J. Demkowicz, A. Misra, and B. P. Uberuaga, Prog. Mater. Sci. 74, 125 (2015).

${ }^{6}$ F. Chen, X. Tang, Y. Yang, H. Huang, and D. Chen, J. Nucl. Mater. 452, 31 (2014).

7J. Luo, P. Tian, C. T. Pan, A. W. Robertson, J. H. Warner, E. W. Hill, and G. A. D. Briggs, ACS Nano 5, 1047 (2011).

${ }^{8}$ M. Cao, X. S. Zhang, W. H. Liu, H. G. Wang, and Y. D. Li, Diamond Relat. Mater. 73, 199 (2017).

${ }^{9}$ J. Wang, Y. Wang, Y. Xu, Y. Zhang, B. Zhang, and W. Wei, Chin. Phys. C 40, 117003 (2016).

${ }^{10} \mathrm{G}$. Xie, W. Cui, and J. Yang, in 17th International Symposium on Antenna Technology and Applied Electromagnetics (ANTEM), Montreal, Canada, 10-13 July 2016.

${ }^{1}$ H. Nguyen, J. Mankowski, J. C. Dickens, A. A. Neuber, and R. P. Joshi, AIP Adv. 8, 015325 (2018).

${ }^{12}$ M. Izawa, Y. Sato, and T. Toyomasu, Phys. Rev. Lett. 74, 5044 (1995).

${ }^{13}$ K. Ohmi, Phys. Rev. Lett. 75, 1526 (1995).

14J. R. M. Vaughan, IEEE Trans. Electron Devices 35, 1172 (1988).

${ }^{15}$ J. Chang, P. Lawless, and T. Yamamoto, IEEE Trans. Plasma Sci. 19, 1152 (1991).

16J. R. M. Vaughan, IRE Trans. Elect. Dev. 8, 302 (1961).

${ }^{17}$ H. C. Kim and J. P. Verboncoeur, Phys. Plasmas 12, 123504 (2005).

${ }^{18}$ R. A. Kishek, Y. Y. Lau, L. K. Ang, A. Valfells, and R. M. Gilgenbach, Phys. Plasmas 5, 2120 (1998).

${ }^{19}$ N. Balcon, D. Payan, M. Belhaj, T. Tondu, and V. Inguimbert, IEEE Trans. Plasma Sci. 40, 282 (2012). 
${ }^{20}$ M. J. Allen, V. C. Tung, and R. B. Kaner, Chem. Rev. 110, 132 (2010).

${ }^{21}$ A. H. Castro Neto, F. Guinea, N. M. R. Peres, K. S. Novoselov, and A. K. Geim, Rev. Mod. Phys. 81, 109 (2009).

${ }^{22}$ A. R. Ranjbartoreh, B. Wang, X. Shen, and G. Wang, J. Appl. Phys. 109 014306 (2011).

${ }^{23} \mathrm{M}$. Nastasi, J. Mayer, and J. Hirvonen, Ion-Solid InteractionsFundamentals and Applications (Cambridge University Press, Cambridge, Great Britain, 1996).

${ }^{24} \mathrm{~J}$. F. Ziegler, J. P. Biersack, and U. Littmark, The Stopping and Range of Ions in Matter (Pergamon, New York, 1985).

25J. F. Ziegler, Nucl. Instrum. Methods Phys. Res. B 219-220, 1027 (2004).

${ }^{26}$ D. J. Bacon, F. Gao, and Y. N. Osetsky, J. Nucl. Mater. 276, 1 (2000) and references therein.

${ }^{27}$ A. V. Krasheninnikov and F. Banhart, Nat. Mater. 6, 723 (2007).

${ }^{28}$ D. B. Graves and P. Brault, J. Phys. D 42, 194011 (2009).

${ }^{29}$ S. Garaj, W. Hubbard, and J. A. Golovchenko, Appl. Phys. Lett. 97, 183103 (2010).

${ }^{30}$ O. Lehtinen, J. Kotakoski, A. V. Krasheninnikov, A. Tolvanen, K. Nordlund, and J. Keinonen, Phys. Rev. B 81, 153401 (2010).

${ }^{31}$ R. J. T. Nicholl, H. J. Conley, N. V. Lavrik, I. Vlassiouk, Y. S. Puzyrev, V. P. Sreenivas, S. T. Pantelides, and K. I. Bolotin, Nat. Commun. 6, 8789 (2015).
${ }^{32}$ K. Saitoh and H. Hayakawa, Phys. Rev. B 81, 115447 (2010).

${ }^{33}$ For example, T. Diaz de la Rubia and G. H. Gilmer, Phys. Rev. Lett. 74, 2507 (1995).

${ }^{34}$ S. Plimpton, J. Comput. Phys. 117, 1 (1995).

${ }^{35}$ M. S. Daw and M. I. Baskes, Phys. Rev. B 29, 6443 (1984).

${ }^{36}$ S. M. Foiles, M. I. Baskes, and M. S. Daw, Phys. Rev. B 33, 7983 (1986).

${ }^{37}$ E. Lennard-Jones, Proc. R. Soc. London A 106, 463 (1924).

${ }^{38}$ Y. Kim, J. Baek, S. Kim, S. Kim, S. Ryu, S. Jeon, and S. M. Han, Sci. Rep. 6, 24785 (2016)

${ }^{39}$ A. V. Sidorenkov, S. V. Kolesnikova, and A. M. Saletsky, Eur. Phys. J. B 89, 220 (2016).

${ }^{40}$ S. P. Huang, D. S. Mainardi, and P. B. Balbuena, Surf. Sci. 545, 163 (2003).

${ }^{41}$ S. J. Stuart, A. B. Tutein, and J. A. Harrison, J. Chem. Phys. 112, 6472 (2000).

${ }^{42}$ Y. N. Osetsky, D. J. Bacon, and B. N. Singh, J. Nucl. Mater. 307-311, 866 (2002).

${ }^{43}$ M. T. Robinson, Defects and Radiation Damage in Metals (Cambridge University Press, Cambridge, 1969).

${ }^{44}$ W. Setyawan, A. P. Selby, N. Juslin, R. E. Stoller, B. D. Wirth, and R. J. Kurtz, J. Phys.: Condens. Matter 27, 225402 (2015).

${ }^{45}$ C. Herbig and T. Michely, 2D Mater. 3, 025032 (2016). 